\title{
IV. Bilanz
}

Die Entnazifizierung in Mecklenburg war 1948 nicht zu Ende. Viele ihrer zentralen Elemente wie die Internierungslager, die 201-Strafkammern, die Kontrollratsdirektive Nr. 38 oder K- 5 blieben in der Sowjetischen Besatzungzone länger bestehen als die Entnazifizierungskommissionen. Der in dieser Studie vorgenommene Einschnitt orientiert sich an dem offiziellen Abschluß der Entnazifizierung und dem weitgehenden Verlust der landespolitischen Selbständigkeit in diesem Bereich. Das von der Besatzungsmacht verkündete Ende war aber mehr als nur Propaganda. Seitdem hatten politische Überprüfungen in der SBZ noch weniger als bisher mit der Zeit der NS-Herrschaft zu tun. Mit dem offiziellen Entnazifizierungsende war die bereits 1945 einsetzende Entwicklung abgeschlossen, die personalpolitischen Überprüfungen immer weniger am Verhalten während des ,Dritten Reiches“ zu orientieren. Sie glichen sich den auch in anderen, Volksdemokratien und kommunistischen Parteien als Herrschaftstechnik üblichen, Säuberungen' nun fast vollständig an. Der Konflikt zwischen dem christdemokratischen Wirtschaftsminister Siegfried Witte und dem kommunistischen Innenminister Johannes Warnke belegt zudem, daß die Innenpolitik in der SBZ 1948 nicht mehr in den Landeshauptstädten, sondern von der Deutschen Verwaltung des Innern (DVdI) in Berlin aus zentral bestimmt wurde.

In der vorliegenden Arbeit wird die schnelle Übernahme politischer Herrschaft durch eine kleine Gruppe von Kommunisten beschrieben. Der Untersuchungsschwerpunkt lag bei der Verwaltung und ihrer Umgestaltung mit Hilfe der Entnazifizierungspolitik. Das erste Kapitel behandelt die rasche und durchgreifende Entnazifizierung, mit der ein tiefgreifender Bruch in der Verwaltungstradition auf Stadt-, Kreis- und Landesebene eintrat. Das zweite Kapitel rekonstruiert zuerst den Aufbau eines neuen Verwaltungstyps, den vor allem seine Unterordnung unter die hegemoniale politische Partei auszeichnete, und danach die Veränderung des Antifaschismus, der zunehmend stärker seiner Bezüge zur NS-Vergangenheit entledigt und seitens der KPD/SED zu einer allgemeinen Integrationsideologie für die Bevölkerung umfunktioniert wurde. Anhand der neu aufgelegten Entnazifizierung zeigt sich im dritten Kapitel noch deutlicher als zuvor, daß die Überprüfungen und Entlassungen niemals in erster Linie auf individuelle Belastungsmomente zielten, sondern stets als Instrument zur Durchsetzung darüber hinausgehender politischer Ziele dienten.

Unmittelbar nach dem Einmarsch der Roten Armee im Mai 1945 waren politische Instrukteure der von dem kommunistischen Remigranten Gustav Sobottka geleiteten Initiativgruppe nach Mecklenburg und Vorpommern gekommen, die gemeinsam mit den sowjetischen Kommandanturen für die Reaktivierung des lokalen öffentlichen Lebens sorgen sollten. Sie nahmen Kontakt zu den örtlichen Antifa-Ausschüssen auf, in denen sich NS-Gegner verschiedener politischer Herkunft bei Kriegsende organisiert hatten. Dabei richteten sich die Instrukteure nach den Weisungen, die sie noch im sowjetischen Exil erhalten hatten. Die Entnazifizierung bildete keinen Schwerpunkt ihrer Aktivitäten: Die am schwersten belasteten Nazis waren bereits geflohen, untergetaucht oder von deutschen NS-Gegnern abgesetzt worden. Neben der Lösung der dringendsten ökono- 
mischen, sozialen und infrastrukturellen Probleme betrieben die Remigranten und ehemaligen Kriegsgefangenen vor allem die Besetzung der vakanten Stellen an der Spitze der lokalen Verwaltungen mit zuverlässigen und ihnen ergebenen Personen. Dabei konnten sie im Norden der SBZ nur auf wenige Kommunisten, Sozialdemokraten und andere parteipolitisch erfahrene NS-Gegner zurückgreifen. Mecklenburg und Vorpommern waren im Gegensatz zu den südlichen Ländern der SBZ überwiegend großagrarisch geprägt. Es gab weder ein starkes Bürgertum noch eine starke Sozialdemokratie als mögliche Träger einer demokratischen und partizipativen politischen Kultur. Dieser Mangel, der durch das traditionell niedrige Bildungsniveau verschärft wurde, erleichterte es der Besatzungsmacht und KPD, die eigene politische Vormachtstellung schnell durchzusetzen - schneller als in den anderen Ländern und Provinzen der SBZ, wo die KPD/SED-Vertreter in Verwaltung und Regierung mit stärkerer Gegenwehr zu kämpfen hatten als die Schweriner Gruppe um Warnke. Aufgrund des dünnen Personalreservoirs war dies nicht nur die Stunde zahlreicher zwielichtiger, auch krimineller Charaktere, die oft bereits nach kurzer Zeit wieder abgesetzt wurden, sondern vor allem vieler Personen aus Arbeiter- und Bauernfamilien, die durch das Einrücken in politische Ämter zu den primären Nutznießern der zuerst von Niethammer beschriebenen außerordentlichen sozialen Aufstiegsmobilität in der SBZ und frühen DDR wurden.

Bevor die sowjetische Besatzungsmacht am 1. Juli 1945 West-Mecklenburg und andere westliche Gebiete der SBZ gegen Westberlin eingetauscht und wenige Tage später das Land Mecklenburg-Vorpommern, seine Landesverwaltung und die Sowjetische Militäradministration Mecklenburgs (SMAM) gegründet hatte, spielte sich das gesamte politische Leben - und damit auch die Entnazifizierung - auf der Ebene der Kreise und Gemeinden ab. Am Beispiel des erweiterten Kreises Güstrow hat die vorliegende Arbeit gezeigt, wie eine neue und nur durch die Siegermacht legitimierte Verwaltung etabliert und in wenigen Wochen auf eine einzige, mit umfangreicher Macht ausgestattete Person, nämlich auf den Oberbürgermeister und späteren Innenminister Warnke, ausgerichtet wurde. Nicht in allen Kreisen und Städten setzte sich die KPD so schnell und erfolgreich durch wie in Güstrow. In Stralsund und Rostock zeigte sich aber ebenso wie in anderen Städten und Kreisen, daß eine stärkere Sozialdemokratie bald durch das Zusammenwirken von kommunistischer Partei und kommunistischer Besatzungsmacht geschwächt wurde.

Welche Bedeutung die Kommunisten strukturellen Veränderungen und Zugriffsmöglichkeiten zumaßen, zeigte sich schon bei dem ersten Konflikt innerhalb des Präsidialkollegiums. Bereits wenige Wochen nach der Installierung der Landesverwaltung und der SMAM in Schwerin konkurrierten verschiedene Politiker um den entscheidenden Einfluß auf die Personalpolitik, die zunächst von der Präsidialabteilung des sozialdemokratischen Landes- und späteren Ministerpräsidenten Wilhelm Höcker verantwortet wurde. Diese Zuständigkeit wechselte Anfang August 1945 gemäß kommunistischer Richtlinie von dem alten, seit der Weimarer Republik im mecklenburgischen Staatsdienst bewährten Verwaltungsfachmann der Präsidialabteilung Hans Jeß zu der von Warnke geleiteten Abteilung Innere Verwaltung. Die Entscheidungsfindung weist auf den direkten Einfluß der Besatzungsmacht hin, ohne daß dieser anhand der deutschen Quellenüberlieferung nachweisbar wäre; für den gesamten Untersuchungszeitraum belegen Hinweise im Schriftgut der deutschen Politiker die sowjetische Praxis, Befehle und Anweisungen fast ausschließlich mündlich zu erteilen. Seit August 1945 zeichnete 
Warnkes Abteilung dann fast allein für die gesamte Personal- und damit auch Entnazifizierungspolitik in Mecklenburg-Vorpommern verantwortlich. Dies änderte sich erst im Spätsommer 1947, als die DVdI im Zuge des SMAD-Befehls Nr. 201 in dieser Frage zunehmend stärker in Landesangelegenheiten intervenierte. Seitdem erhielt Warnke seine Weisungen nicht mehr von der SMAM, sondern von dieser Zentralverwaltung in Berlin.

Zwei Monate nach Gründung der Landesverwaltung und der SMAM wurde die Entnazifizierung ebenso wie die Sequestrierungspolitik landesweit systematisiert. Damit entzog Warnkes Abteilung die Personalpolitik den Kreisen, Städten und ihren lokalen Funktionsträgern und übertrug sie dem zentralen Personalamt auf Landes-, Kreis- und Stadtebene. Bis zum November 1945 - schneller und rigoroser als in anderen Ländern der SBZ - hatte die Landesverwaltung in Mecklenburg-Vorpommern die Entlassung sämtlicher Verwaltungsangehöriger durchgeführt, die der NSDAP oder ihren Unterorganisationen angehört hatten. Für den ersten umfassenden Entlassungsschub legte die Abteilung Innere Verwaltung Kriterien fest, die ausdrücklich weiter gefaßt waren als die entsprechenden Beschlüsse der Potsdamer Konferenz und des Zentralen Parteienblocks in Berlin. Nur wenige Wochen später ordnete die SMAD auf einer Konferenz der Landespräsidenten weitere Entlassungen an, durch die die Entnazifizierung in der öffentlichen Verwaltung vorerst beendet wurde. Wiederum zeichnete sich Mecklenburg-Vorpommern durch einen besonderen Rigorismus aus; früher als die anderen Länder konnte es die Erfüllung der umfangreichen Entlassungsforderung melden.

1945 wurde das Hauptaugenmerk bei der Entnazifizierung auf die Entlassungen und Neueinstellungen in den Verwaltungen des Landes, der Kreise und Städte gelegt, da sie die organisatorische Grundlage der Landespolitik bildeten. Bereits in den ersten Wochen nach Kriegsende hatte die Besatzungsmacht sämtliche Polizisten entlassen, teilweise interniert und durch Mitglieder der beiden Arbeiterparteien ersetzt. Auch die Entnazifizierung der Juristen und Lehrer ging schnell voran: Ebenso wie in der Verwaltung gab es bereits Ende 1945 keine ehemaligen NSDAP-Mitglieder mehr im mecklenburgischen Justizwesen; Anfang Januar 1946 erließ die Landesverwaltung für die Lehrerschaft eine ähnlich rigorose Entnazifizierungsverordnung, wie sie für die Verwaltungsmitarbeiter bestand. Mediziner, Mitarbeiter von Post und Bahn und andere Berufsgruppen, auf deren Leistungen aus gesellschaftlichen oder politischen Gründen kaum verzichtet werden konnte, behandelte die Landesverwaltung dagegen vorerst viel zurückhaltender.

Diese Zurückhaltung resultierte aber auch daraus, daß die KPD nur bei bestimmten Personen- und Berufsgruppen sowie Institutionen einen so tiefen Bruch intendierte, wie sie ihn mit Hilfe der Entnazifizierung in den staatlichen Verwaltungen durchsetzte. Beispielhaft dafür ist die Wirtschaft: Die Entnazifizierung der privaten Unternehmen wurde früh, auf lokaler Ebene und häufig betriebsintern durchgeführt. Überprüfungskommissionen setzten bereits bei Kriegsende die Entlassung oder Degradierung der am schwersten und offensichtlichsten NS-belasteten Mitarbeiter durch. Ende August 1945 begann die Landesverwaltung, nicht nur die Entnazifizierung der Verwaltung, sondern auch die Sequestrierungspolitik der Verantwortung der Kreise und Städte zu entziehen und sie landesweit zu vereinheitlichen. Seitdem vernachlässigte sie bei der politischen Kontrolle der Betriebe die Überprüfungen und Entlassungen einzelner Angestellter und Arbeiter; statt dessen konzentrierte sie sich auf die Betriebsinhaber und auf Möglichkeiten, ihr Eigentum in treuhänderische Verwaltung zu überführen. Aufgrund der länderübergreifenden Vernetzung großer Unternehmen und des Interesses der Besatzungs- 
macht an Reparationen und einer die Selbstversorgung der SBZ sichernden wirtschaftlichen Gesundung vereinheitlichten sowjetische Befehle die ,politische Bereinigung der Wirtschaft bereits im Oktober 1945 zonenweit. Nunmehr verloren diese Verfahren ihren personalpolitischen Charakter vollständig zugunsten des wirtschaftspolitischen; die Entnazifizierung der Betriebe im Sinne einer Personalüberprüfung wurde erst ein Jahr später wieder aufgenommen.

Die Ingangsetzung der Produktion, die umfangreichen Sequestrierungen und andere wirtschaftspolitische Ziele waren wichtiger als die Entnazifizierung. Während die Landesverwaltung die Entnazifizierung des öffentlichen Dienstes ausdrücklich gegen sämtliche, selbst subalterne Mitarbeiter richtete, wurden die Entlassungen aus Wirtschaftsunternehmen nach den ersten Monaten der Besatzung eingestellt. Viele in der Wirtschaft tätige ehemalige Mitglieder von NS-Organisationen entkamen dadurch 1945 der Entnazifizierung.

Ähnlich erging es den Flüchtlingen. Sie blieben von der Entnazifizierung weitgehend ausgenommen. Mecklenburg-Vorpommern hatte mehr Flüchtlinge aus den ehemaligen deutschen Ostgebieten aufzunehmen als alle anderen Länder und Provinzen Deutschlands. Dadurch erfaßte die Entnazifizierungspolitik dort nur etwas mehr als die Hälfte der erwachsenen Bevölkerung. Zuerst resultierte dies aus verschiedenen organisatorischen Schwierigkeiten: 1945 gab es trotz der Registrierungsversuche in Städten, Kreisen und auf Landesebene keinen Überblick über die stark schwankende Bevölkerungszahl; personenbezogene Unterlagen über die einzelnen Flüchtlinge waren nicht vorhanden; die Zerstörung der alten sozialen Netze durch die Flucht und der aus gemeinsam erlebter Not erwachsene Zusammenhalt hatten zur Folge, daß es seltener zu Anzeigen und Denunziationen durch ehemalige Nachbarn, Kollegen oder Verwandte kam als unter der alteingesessenen Einwohnerschaft Mecklenburg-Vorpommerns, deren soziale Beziehungen mindestens partiell erhalten gebliebenen waren. Als 1946 die Überprüfung der Flüchtlinge aufgrund der in München aufgefundenen NSDAP-Mitgliederkartei möglich wurde, unternahm die Landesregierung keine entsprechenden Anstrengungen, da sie die Entnazifizierung Ende 1945 bereits für beendet erklärt hatte. Dies zeigt den Pragmatismus, der 1948 auch das Ende der zweiten Entnazifizierungswelle in der SBZ auszeichnete und der mit dem großen Interesse an einem reibungslosen Neuaufbau der Verwaltung verbunden war. Bei der Besetzung der vakant gewordenen Stellen mit Flüchtlingen interessierte weniger ihre NS-Vergangenheit als die Bereitschaft zur aktiven und politisch loyalen Aufbauarbeit. Für die Umsiedler bedeutete das bereits 1945 das Ende der Entnazifizierung, die 1948 endgültig eingestellt wurde. Die langsame gesellschaftliche Reintegration wurde zur Gegenleistung für politische Loyalität.

Die ungleiche Intensität der Entnazifizierung zeigt das über die politische Überprüfung hinausgehende Interesse an der Entnazifizierung seitens der Landesverwaltung. Entscheidend war nicht die Vergangenheit der jeweiligen Einzelpersonen, sondern der gesellschaftliche Neuanfang. Verwaltungspolitisch verfolgten KPD und Besatzungsmacht dabei das Ziel, die neue staatliche Bürokratie in der Tradition leninistischer Parteiapparate als ,Kaderverwaltung (Balla) zu errichten. Daher wurden die neuen Verwaltungsdienstkräfte und die wenigen in ihren Stellen Verbliebenen bereits seit dem ersten Besatzungsjahr dahingehend geschult, jeden Verwaltungsakt durch übergeordnete politische ,Notwendigkeiten“ aufheben oder verändern zu lassen. Die Definition dessen, was politisch ,notwendig' war, oblag der KPD/SED oder der Besatzungsmacht, die ihre ,Kompetenzkompetenz“ (Lepsius) damit in der Verwaltung noch schneller durchsetzen 
konnten als in anderen gesellschaftlichen Bereichen wie der Wirtschaft, der Kulturpolitik oder der Justiz.

Der Aufbau der Kaderverwaltung mit ihren spezifischen politischen Anforderungen ging mit dem Ausbau einer Monopolstellung des Personalamts einher. Neben der Schulung und Auswahl der neuen Dienstkräfte bemühte sich dieses Amt besonders um den Ausbau und die Festigung der eigenen Machtposition. Es zentralisierte die Personalpolitik, indem es sich das Informationsmonopol für die Personalangelegenheiten sämtlicher staatlicher Dienstkräfte in Mecklenburg-Vorpommern und gleichzeitig umfangreiche formale Eingriffsmöglichkeiten bei allen Einstellungen und Entlassungen sicherte. Im Laufe dieses Prozesses übernahm ein kommunistischer Remigrant aus der Sowjetunion, Erich Kundermann, die Leitung der Behörde, die ihren Einfluß trotz diverser Kontroversen mit Landespräsident Höcker und dem Präsidialkollegium durchsetzen konnte. Damit war Warnkes Vormachtstellung gegenüber Höcker und den anderen Vizepräsidenten so eindeutig abgesichert, daß der Landespräsident - anders als beispielsweise Rudolf Paul in Thüringen - seitdem keine bedeutsame politische Angelegenheit mehr entscheidend zu beeinflussen vermochte. Die so gewonnene Vorrangstellung war die politische und organisatorische Ausgangsbasis des Personalamtes für die vom Herbst 1946 bis zum Frühjahr 1948 wiederaufgenommene Entnazifizierung sowie für die anschließenden Disziplinierungen, Überprüfungen und ,Säuberungen‘.

Die Veränderungen in der staatlichen Bürokratie spiegelten sich auch in den fachlichen, politischen und sozialen Anforderungen an das neue Personal: Dessen Auswahl orientierte sich weniger an der fachlichen Qualifikation als an der sozialen Herkunft und der politischen Bindung. Das Landespersonalamt bevorzugte die Einstellung von Arbeitern, Bauern und ihren Familienangehörigen sowie von Mitgliedern der Blockparteien. Außerdem richtete sich die politische Aufmerksamkeit nach dem Ende der Entnazifizierung 1945/46 zunehmend weniger auf NS-Belastungen als auf die Loyalität zur Besatzungsmacht und zur KPD/SED. Diese Verschiebung des Belastungsprofils trieb die von der KPD bereits im Exil und in den ersten Besatzungsmonaten vorbereitete gesellschaftliche Reintegration der angeblich bloß nominellen Nazis voran und entledigte den Faschismusvorwurf zunehmend seiner Bezüge auf die Zeit des,Dritten Reiches‘. Der Antifaschismus wurde statt dessen - wie ihn die KPD schon in der Weimarer Republik verstanden hatte - zur Freund-Feind-Chiffre und von der SED schon seit Beginn des Kalten Krieges zur Polarisierung zwischen Ost- und Westdeutschland aktiviert.

Dieser Verschiebung von Antifaschismus und Faschismusvorwurf entsprach ein besonderer Umgang mit den Opfern und Tätern der NS-Herrschaft. Schon 1945 zeigte sich, daß die KPD/SED die Opfer des ,Dritten Reiches' fast ausschließlich unter dem Gesichtspunkt des eigenen politischen Kampfes wahrnahm. Dementsprechend hing die Förderung oder Ausgrenzung nichtkommunistischer Opfer- und Widerstandsgruppen in der SBZ davon ab, ob sie gerade ins politische Konzept der Kommunisten paßten. Schon mehrere Jahre bevor in Mecklenburg ein Entschädigungsgesetz zugunsten der Opfer des Faschismus verabschiedet wurde, stand bereits die Werbung um die ehemaligen NSDAP-Mitglieder auf der Agenda der KPD und der anderen Parteien. Davon profitierten beide Seiten: Die Parteien gewannen Mitglieder, Wähler und treue Untertanen, und die Pgs konnten sich bei ausreichender Anpassungsbereitschaft politisch und moralisch salvieren, da das Bekenntnis zu den einzig ,wahrhaft antifaschistischen Mächten', Besatzungsmacht und KPD, als Ausweis praktischer Umkehr und Umerziehung galt. 
Im Laufe des Jahres 1946 erwies sich, daß das schnelle Ende der Entnazifizierung den außenpolitischen Interessen der Sowjetunion widersprach. Ebenso wie die westlichen Besatzungsmächte bereitete sie sich auf eine Konferenz der alliierten Außenminister im Frühjahr 1947 vor, bei der gegenseitige Vorhaltungen über eine unzureichende Entnazifizierung in der jeweils anderen Zone eine nicht unerhebliche Rolle spielen sollten. Daher veranlaßte die SMAD einige Monate vor der Konferenz die Wiederaufnahme der Entnazifizierung. In Ländern, Kreisen und Gemeinden sollten nun nach der bereits im Januar 1946 verabschiedeten Alliierten Kontrollratsdirektive Nr. 24 Entnazifizierungskommissionen errichtet werden. Die weitgehende Passivität der Kommissionen und des verantwortlichen Personalamtes werfen ein deutliches Licht auf die fast ausschließlich außenpolitische Funktion dieser Neuauflage der Entnazifizierung; die Kreise und Städte, die Abteilung Innere Verwaltung und die SMAM zeigten sich an erneuten Überprüfungen und Entlassungen wenig interessiert. Den Sowjets genügte offenbar der äußere Schein, für die SED hatte das reibungslose Funktionieren der Verwaltung Vorrang, und auch in der Bevölkerung stieß die Entnazifizierung auf immer weniger Zustimmung und Verständnis. Die besonders in der westlichen Forschung hervorgehobene Bedeutung der Gemeinde- und Landtagswahlen in der SBZ im Herbst 1946 muß dagegen relativiert werden. Wahlkampfstrategische Erwägungen der SED spielten für den Zeitpunkt der Wiederaufnahme der Entnazifizierung eine nur untergeordnete Rolle, denn die Kreiskommissionen wurden in Mecklenburg-Vorpommern bereits vor diesen Wahlen eingerichtet.

Die Anwendung der Kontrollratsdirektive Nr. 24 fiel nicht leicht, da die meisten Pgs bereits seit 1945 aus dem öffentlichen Dienst entlassen worden waren. Darum standen vor den Kommissionen hauptsächlich Personen, die als Selbständige, Arbeiter oder Angestellte in der privaten Wirtschaft arbeiteten. Indessen hatte die zweite Welle der Entnazifizierung eine breit gestreute disziplinierende Wirkung, da die Mitarbeiter sämtlicher Betriebe, Organisationen und Behörden zu einer detaillierten Offenbarung ihrer politischen Vergangenheit verpflichtet wurden, bei der jeder mit Unterschrift seine Unschuld und politische Loyalität bekunden mußte. Dabei ging es zunehmend weniger um NSBelastungen als um einen weiter gefaßten Faschismusvorwurf: Die alliierte Entnazifizierungsdirektive bot einen so weiten Interpretationsspielraum, daß kommunistischer Antifaschismus und kontrollratsgemäße Entnazifizierung in der SBZ zu Synonymen wurden. Der Alliierte Kontrollrat hatte eine umfangreiche Liste von Kriterien zusammengestellt, nach der Belastete gesucht und ihre Entlassung ermöglicht oder vorgeschrieben wurde. Der Umfang und die Ungenauigkeit dieser Bestimmungen ermöglichten es, fast jede individuelle Verfolgung von politischen Gegnern als Teil der Entnazifizierung zu rechtfertigen. Dies stärkte den kommunistischen Dualismus von Faschismus und Antifaschismus und reproduzierte das dichotome Weltbild der KPD.

Offiziell blieben die Entnazifizierungskommissionen mehrere Monate bestehen, obwohl die meisten ihre Überprüfungstätigkeit bereits früher eingestellt hatten. Sie konnten sich nicht offiziell auflösen, hatten aber auch kein klares Ziel mehr. Dieser Latenzzustand war ein Unsicherheitsfaktor und eine beständige politische Bedrohung für alle abhängig Beschäftigten. Die Entnazifizierung rief daher in der Bevölkerung eine allgemeine Unzufriedenheit hervor. Als selbst die Spitzen der Blockparteien ihr Mißfallen artikuliert hatten, wurde der SMAD-Befehl Nr. 201 erlassen, der ein schnelles Ende der Verfahren versprach. In einem überschaubaren Zeitraum sollten die NS-Belasteten end- 
gültig aus allen Verwaltungen und Leitungspositionen entlassen und die strafrechtlich Schuldigen von eigens dafür eingerichteten Strafkammern verurteilt werden. Während sich diese Strafverfahren über das offizielle Ende der Entnazifizierung hinauszogen, waren alle nicht strafrechtlich Belastbaren nach dem offiziellen Abschluß praktisch amnestiert: In der SBZ sollte danach nicht mehr zwischen aktiven und bloß nominellen Nazis, sondern nur noch zwischen unbelasteten und verbrecherischen Bürgern unterschieden werden. Die erste Begeisterung für diesen Befehl verflog in der Bevölkerung schnell, als offenkundig wurde, daß die SED ihn nicht im Sinne eines sofortigen Abschlusses, sondern einer vorläufigen Forcierung der politischen Überprüfungen nutzte. Die Kommissionen nahmen ihre Tätigkeit für einige Monate wieder auf. Der Befehl Nr. 201 verkörperte also beide Seiten der Entnazifizierungspolitik von SED und SMAD: Einerseits beschleunigte er ihr Ende, andererseits diente er dazu, Institutionen zu installieren, die später zum organisatorischen Kernbereich der paranoiden DDR-Sicherheitspolitik wurden. Die dabei schon 1947/48 zutage tretende Zunahme von Verfahren gegen politische Abweichungen verdeutlicht, wie stark das Interesse der Entnazifizierungsorgane an den eigentlichen Verstrickungen in das NS-Herrschaftssystem abnahm.

Die Entnazifizierung als nicht juristisch, sondern ausschließlich politisch legitimierte Abrechnung mit dem Faschismus fand im Frühjahr 1948 erneut einen geordneten Abschluß. Dieser ermöglichte wiederum - wie Ende 1945 - verstärkte Versuche zur systematischen Reintegration der ehemaligen NSDAP-Mitglieder in die neue Gesellschaft. Die für den Abschluß der Entnazifizierung entscheidenden SMAD-Befehle Nr. 201 vom August 1947 und Nr. 35 vom Februar 1948 enthielten eindeutige Offerten an anpassungsbereite Pgs, die sich im Sinne von Sowjets und SED beim Neuaufbau bewährten. Aufgrund dieser von SMAD und SED offen vertretenen Politik öffneten sich im Laufe der folgenden Jahre für ehemalige Nazis in der DDR zunehmend mehr Möglichkeiten für neue berufliche und gesellschaftliche Karrieren.

Dazu trugen in nicht unerheblichem Maße die Parteiorganisationen bei. Abgesehen von der Delegation eigener Mitglieder in die Kommissionen zeigten sich die Parteien an der Entnazifizierung weitgehend desinteressiert. Sie überließen sie fast vollständig der Verantwortung der Abteilung bzw. dem Ministerium für Innere Verwaltung. Die ehemaligen Pgs weckten das Interesse der Parteien nur, soweit es um ihre Aufnahme als Mitglieder ging. Diese Frage war ein politischer Dauerbrenner, bei dem die Besatzungsmacht generelle Verfahrensentscheidungen verhinderte. Damit standen alle Parteien stets unter einem Legitimationsdruck hinsichtlich der immer wieder in ihren Reihen nachweisbaren ehemaligen Nazis. Diese waren in einer ähnlichen Situation, da sie fortwährend praktisch beweisen mußten, daß sie sich tatsächlich von ihrer NS-Vergangenheit getrennt hatten. Ähnlich wie die anderen neuen Parteien entwickelte sich die SED durch die bis in die fünfziger Jahre hinein zunehmende Anzahl ehemaliger Pgs in ihren Reihen zu einer "Mitläuferpartei neuen Typs“. ${ }^{1}$ Dies belegt den Erfolg der auf Integration zielenden Politik von SED und SMAD: Im Laufe weniger Jahre hatten sich die meisten ehemaligen NSDAP-Mitglieder wiederum erfolgreich zu treuen, diesmal ,volksdemokratischen' Untertanen entwickelt.

\footnotetext{
${ }^{1}$ Foitzik, Säuberungen, S. $414 f$.
} 
Die Anforderungen an die politische Loyalität ließen kein Ende der Überprüfungen zu. Obwohl Warnke sich nicht mit dem Konzept hatte durchsetzen können, den Apparat der Entnazifizierungskommissionen bruchlos in eine Instanz permanenter politischer ,Säuberung zu überführen, wurde die Entnazifizierung trotz ihres offiziellen Endes in verschiedenen Formen fortgesetzt. Damit begann auch eine DDR-Tradition, in der politische Gegensätze nicht ausgeglichen, sondern vor dem Hintergrund der antifaschistischen Interpretation der jeweiligen Gegenwart und jüngsten Vergangenheit verschärft wurden:

Die aufgrund des Befehls Nr. 201 geschaffenen Entnazifizierungsstrafkammern waren zu engster Zusammenarbeit mit der dafür in umfangreichem Maße ausgebauten politischen Polizei (K-5) verpflichtet. Sowohl K-5 als auch die 201-Strafkammern arbeiteten nicht nur die von den Entnazifizierungskommissionen bis zum Frühjahr 1948 übergebenen Fälle ab, sondern setzten ihre Tätigkeit darüber hinaus fort. Während K-5 Teil des DDR-Sicherheitsapparates wurde und bis 1989 im Ministerium für Staatssicherheit bestehenblieb, führten die Strafkammern ihre politisierte Rechtsprechung gegen tatsächliche und vermeintliche, aktive Nazis und Kriegsverbrecher bis Anfang der fünfziger Jahre fort. Die Einrichtung dieser speziellen Entnazifizierungsstrafkammern nutzte die SED in der ganzen SBZ zur forcierten politischen Disziplinierung, Schulung und Zentralisierung der Gerichte und Staatsanwaltschaften. Die in Folge des Befehls Nr. 201 stark veränderten Kompetenzgrenzen zwischen den dafür zuständigen Staatsanwaltschaften und Polizeiorganen zeigt die Funktionalisierung dieser politischen Strafverfahren zum Umbau des Justizsystems nach sowjetischem Muster. Bei den Waldheimer Prozessen wurden ausdrücklich Richter und Staatsanwälte herangezogen, die sich in diesen 201-Verfahren ,bewährt' hatten.

Es zeigen sich noch weitere Verbindungen zwischen der Entnazifizierung und der Staatssicherheitspolitik der DDR. Der seit 1947 für die Entnazifizierung verantwortliche DVdI-Vizepräsident Erich Mielke zog sämtliche personenbezogenen Entnazifizierungsunterlagen in der DVdI in Berlin zusammen, um sich ein ähnliches Informationsmonopol zu sichern, wie es das Personalamt auf Landesebene besaß. Anfang der fünfziger Jahre übernahm das Ministerium für Staatssicherheit nicht nur K-5, sondern auch die gesamten Kommissionsunterlagen. Obwohl diese Akten im Rahmen der Recherchen nach NS-Belasteten anscheinend nur begrenzt ausgewertet wurden, belegt auch diese direkte Kontinuität zwischen der Entnazifizierung und dem Geheim- und Sicherheitsdienst der DDR, wie früh, zentralistisch und umfangreich sich die neuen Herren um eine strukturelle Sicherung ihrer Herrschaft und die Verhinderung politischer Opposition bemühten.

Die fünf Länder und Provinzen hatten zu Beginn der Besatzungszeit eine erhebliche Eigenständigkeit besessen. Anhand der Entnazifizierung läßt sich nachvollziehen, wie die Zentralverwaltungen diese Spielräume bis zur Gründung der DDR systematisch verkleinerten: 1945 wurden die Überprüfungen ohne Beteiligung der Zentralverwaltungen und (anders als im Falle der Sequestrierungen und der Bodenreform) ohne besondere Anteilnahme der zonalen Parteileitungen von der Besatzungsmacht angeleitet und in deutscher Hand ausschließlich als Länderangelegenheit durchgeführt. Dies setzte sich bei der Reaktivierung der Entnazifizierung im Spätsommer 1946 fort, als sich wiederum keine der Berliner Zentralverwaltungen, sondern die Besatzungsmacht an die Länderregierungen wandte. Erst mit dem SMAD-Befehl Nr. 201 vom August 1947 sollten lan- 
desspezifische Differenzen durch die Verantwortlichkeit und Kontrollfunktion von der DVdI und der Deutschen Zentralverwaltung für Justiz (DJV) verhindert werden. Um die Selbständigkeit von Warnke und der Landesregierung zurückzudrängen, konnte sich die DVdI der bereits weitgehend zentralisierten Polizei sowie der Parteidisziplin bedienen, da sich in den Landesinnenministerien und der DVdI fast ausschließlich SED-Genossen begegneten. Die Zentralisierung der Entnazifizierung diente der DVdI als Einfallstor zur Einflußnahme auf die Personalpolitik der Landesregierungen. So wie die Entnazifizierung 1945 dazu beigetragen hatte, die monopolartige Stellung des Landespersonalamtes aufzubauen, nutzten die Kommunisten sie auch bei ihrem Auslaufen 1947, um die politischen Strukturen in der SBZ noch weiter zu zentralisieren, die Kontrolle über das staatliche Verwaltungspersonal effektiver zu gestalten und damit die inzwischen seit fast drei Jahren etablierte Herrschaft weiter abzusichern.

Die bis zum Befehl Nr. 201 für die Entnazifizierung verantwortlichen deutschen Stellen waren ausschließlich auf Landes-, Kreis- und Gemeindeebene angesiedelt; erst durch diesen Befehl kamen auch Berliner Stellen ins Spiel. Dabei ist - vor allem gegen die schnelle Apostrophierung der gesamten Geschichte von SBZ und DDR als SED-Diktatur - jeweils zwischen staatlichen und SED-Stellen zu unterscheiden:

Auf zonaler Ebene bestimmte die SED die Politik der quasi-staatlichen Zentralverwaltungen entscheidend; besonders in der DVdI gewann sie seit deren Gründung einen großen Einfluß, da die meisten Mitarbeiter und alle (Vize-)Präsidenten langjährige Kommunisten waren. Eine genaue Differenzierung zwischen den Zentralverwaltungen und dem Zentralsekretariat der SED ist häufig nicht möglich; beispielhaft für die Verquickung beider sind die zonalen Konferenzen der Landesinnenminister, die sämtlich der SED angehörten und auf denen sie von den Vertretern des Zentralsekretariats primär als Genossen und nicht als Träger staatlicher Gewalt angesprochen wurden. Diese Überschneidung zeigt sich auch ex negativo: Bevor sich die Zentralverwaltungen im Rahmen der Entnazifizierung engagierten, zeigten auch das Zentralkomitee der KPD und das Zentralsekretariat der SED nur wenig Interesse an den personalpolitischen Überprüfungen, so daß ihre diesbezüglichen Stellungnahmen von Fehlinformationen und unnützen, weil verspäteten Anordnungen geprägt waren.

Auf Landesebene trat die SED im Rahmen der Entnazifizierung dagegen genauso wenig in Erscheinung wie die anderen Parteien. Sie überließ die Verantwortung ihrem Genossen Warnke, der die ausdrückliche Unterstützung der SMAM hatte und dessen rigorose Politik in seiner Partei keine Kritik hervorrief. Das Landespersonalamt lenkte die Personalpolitik in der Landesverwaltung stellvertretend für die SED und in ihrem Interesse. Eingriffsversuche und zu eindringliche Fragen seitens der Landesleitung der SED wehrte Warnke allerdings souverän ab. Gleichzeitig beanspruchte er keinen bedeutenden Einfluß innerhalb der nur langsam aufgebauten Landespartei.

Die Beurteilung des Verhältnisses von staatlicher Verwaltung und KPD/SED auf Kreisebene bedarf weiterer Lokalstudien; nach den bisherigen Quellenkenntnissen spielte die Differenz zwischen staatlicher Verwaltung und KPD/SED dort eine ähnlich geringe Rolle wie auf zonaler Ebene, weil die Oberbürgermeister und Landräte meistens der SED angehörten. Dabei bewährten sich die Prinzipien der Kaderverwaltung: die Verordnungen und Aufträge der Abteilung Innere Verwaltung trugen stets Befehlscharakter, und expliziter Widerspruch wurde nicht geduldet. Zudem vermischte sich die Arbeit der Verwaltungen mit der der SED und der Blockausschüsse auf lokaler Ebene stär- 
ker als auf Landesebene, da sie in weiten Teilen auf das ehrenamtliche Engagement der Parteimitglieder zur politischen Mobilisierung und organisatorischen Absicherung ihrer Politik angewiesen waren.

Erst kurz vor dem Ende der Entnazifizierung beklagten einige führende Mitglieder der mecklenburgischen CDU und auch der LDP die strukturelle Willkür, die mit der Ausweitung des Faschismusvorwurfs und mit der seit dem Befehl Nr. 201 veränderten Entnazifizierungsstruktur verbunden war. Daher versuchten sie den Landtag - gemäß dem Wortlaut einer sowjetischen Ausführungsbestimmung zum SMAD-Befehl Nr. 201 - als Kontroll- und Korrektivinstanz gegen die Exekutive zu stärken. Weil die Kritik erst nach der Übergabe der Entnazifizierungskompetenz an die DVdI und DJV in Berlin vorgetragen wurde, konnte die Landesregierung keine eigene Entscheidung treffen und mußte die Beantwortung der Anfragen über mehrere Monate hinauszögern. Dies offenbarte den Verlust von Warnkes politischer Selbständigkeit. Erst als DVdI und SMAD ihren Entschluß mitgeteilt hatten, wurde seine Position durchsetzungsfähig.

Ebenso wie der in zahlreichen weiteren Fragen praktizierte enge Schulterschluß der Landesleitungen von CDU und LDP mit der SED drängte auch diese Zurückweisung die kritischen Kräfte in den bürgerlichen Parteien noch weiter ins politische Abseits. Diejenigen, die ihre Ablehnung dieser Entnazifizierungspolitik vorgetragen hatten, wurden zur Zielscheibe der SED-Kritik und zogen sich bald aus der Politik in der SBZ zurück; die meisten gingen in den Westen. Dies zeigt wiederum, wie eindeutig sich die Verfolgungskriterien und die Anforderungen an eine ausreichende politische Loyalität gewandelt hatten; gleichzeitig bestätigt die Erfolglosigkeit der von Witte und anderen seit 1947 formulierten Kritik an der fast ausschließlich SED-bestimmten Landespolitik die These, daß Mecklenburgs Politik und Verwaltung bereits wenige Jahre nach dem Zusammenbruch des, Dritten Reiches' weitgehend unter der Hegemonie der SED standen und daß die von der KPD im Frühsommer 1945 in den Städten und Kreisen begonnene Zentralisierung inzwischen auch gesamtzonal erfolgreich durchgesetzt war.

Die Ergebnisse der vorliegenden Studie zeigen die übergeordneten Ziele, an denen die KPD/SED ihre gesamte Politik schon seit 1945 ausrichtete: Es ging ihr um die Errichtung der eigenen Herrschaft, um gesellschaftliche Strukturveränderungen und um die Zurückdrängung oppositioneller oder gar widerständiger politischer Bestrebungen. Besonders deutliche Beispiele dafür, daß diesen Zielen andere politische Inhalte untergeordnet und geopfert wurden, sind Entnazifizierung und Antifaschismus. Mehrere Hinweise belegten schon 1945 das über die politische Überprüfung hinausgehende Interesse an der Entnazifizierung: erstens der Rigorismus bei den Entlassungen in der Verwaltung, zweitens die Versuche, jegliche Rückkehr eventuell unrechtmäßig Entlassener zu verhindern, drittens die Geschwindigkeit, mit der die Entnazifizierung vollzogen wurde, wodurch neues Personal eingesetzt werden konnte, und viertens das Desinteresse an einer Überprüfung der Flüchtlinge. Es ging ganz offensichtlich nicht um die Beurteilung und Ahndung von einzelnen Belasteten, sondern um eine strukturelle Veränderung: Durch einen breit angelegten Personalwechsel sollte die Bürokratie ausgetauscht werden, die sich seit dem Kaiserreich als vermeintlich, unpolitische ohne große Brüche gehalten und sich selbst im ,Dritten Reich“ als dienstbar erwiesen hatte. Die Entnazifizierung war ein effektives Instrument, das die Kommunisten unabhängig von individuellen Belastungsmomenten gegen die fortgesetzte Herrschaft des bisherigen admini- 
strativen Apparates einsetzten. Die Geschichte des NS-Regimes wurde mitsamt ihren konkreten Tätern und Opfern verdrängt und zur dramatischen Kulisse, vor der gegenwärtige politische Konflikte ausgetragen wurden. Ihre Geschichte von Exil und Widerstand, die die Kommunisten im Frühsommer 1945 ins Zentrum der Legitimation ihrer Führungsposition gerückt hatten, verflüchtigte sich damit bald entweder zu ritualisierten Floskeln oder zu legitimatorischen Stereotypen. Die Entnazifizierung diente als Ersatzlegitimation und der Antifaschismus als Ersatzideologie der revolutionären Veränderungen, weil der Marxismus-Leninismus aufgrund der Volksfrontstrategie tabuisiert war und zudem außerhalb der Gemeinde der sowieso schon überzeugten Kommunisten kaum Glauben fand.

Weil die Gesellschaft der SBZ/DDR unter dem Aspekt der politischen Herrschaft nicht ausreichend erfaßt wird, fragt die neue SBZ/DDR-Forschung nach Frei- und Spielräumen in Gesellschaft und Politik. In dieser Hinsicht liefert die vorliegende Arbeit nur wenige Ergebnisse: Augenscheinlich gab es keinen „kommunistische[n] Meisterplan",2 mit dem Besatzungsmacht und KPD/SED sämtliche Lebensbereiche erfaßten. Ganz offensichtlich zeichnete sich aber das Ziel grundlegender gesellschaftlicher Umgestaltung ab, das schon 1945 weit über die Abrechnung mit NS-Belasteten hinausreichte. Der damit verbundene Rigorismus und die Durchschlagskraft der kommunistischen Politik zeigen sich anhand der staatlichen Verwaltung in Mecklenburg besonders deutlich. Dafür waren vor allem zwei Faktoren verantwortlich: Erstens behandelten KPD/SED und Besatzungsmacht die Verwaltung als zentrales Instrument für die weitere politische Durchdringung der Gesellschaft mit einer größeren Aufmerksamkeit als machtpolitisch weniger wichtige Bereiche wie Kultur, Sport oder Wohlfahrtspolitik. Zweitens führten die regionalen Besonderheiten Mecklenburg-Vorpommerns dazu, daß die Politiker in Schwerin die föderale Struktur der SBZ und die von der Besatzungsmacht gewährten landespolitischen Spielräume ausschöpften.

Der Bruch zeigte sich in Mecklenburg-Vorpommern bereits vor dem Ende des Jahres 1945 in aller Deutlichkeit. Er erfaßte die machtpolitisch zentralen Bereiche, keineswegs aber sämtliche Sektoren der Gesellschaft. Für deren Erforschung bleiben weitere thematische und zeitliche Feinabstimmungen notwendig. Darüber hinaus verweisen die taktischen Windungen und zeitlichen Verzögerungen, mit denen das Wirtschaftsministerium 1947/48 die kommunistische Umgestaltungspolitik behindern konnte, auf eine erheblich niedrigere Durchschlagskraft der politischen Institutionen als noch im ersten Jahr nach Kriegsende bei Bodenreform, Sequestrierung und dem Verwaltungsumbau. Dennoch: Die Entnazifizierung war abgeschlossen, und die Verantwortungsträger richteten ihr Augenmerk auf die Zukunft; die Veränderungen dienten nicht mehr der Abrechnung mit der NS-Vergangenheit, sondern dem Aufbau einer, Volksdemokratie'. Seitdem hielten Kommunisten die Verwaltung in den Händen und duldeten auf landespolitischer Ebene keinen organisierten politischen Widerspruch. Sie monopolisierten den Staatsapparat und bestimmten dank der Schwäche der weiteren Parteien die Landespolitik. Damit standen ihnen die zentralen Instrumente zur Durchdringung der gesamten Gesell-

${ }^{2}$ Jessen, Gesellschaft, S. 99. 
schaft zur Verfügung: Infolge der schnellen und erfolgreichen ,Privatisierung des Staates $^{{ }^{3}}$ durch wenige kommunistische Landespolitiker eilte Mecklenburg-Vorpommern in großen Schritten in die ,durchherrschte Gesellschaft ${ }^{\text {' }}{ }^{4}$

Viele Entwicklungen in Mecklenburg-Vorpommern glichen denen in Brandenburg, Sachsen, Sachsen-Anhalt und Thüringen. Nicht bei generellen Richtungsentscheidungen, wohl aber bei der konkreten Durchführung zeigen sich auch landesspezifische Eigenheiten. Diese markieren eine Tendenz, die Mecklenburg-Vorpommern nicht nur hinsichtlich der Entnazifizierung und des Verwaltungsumbaus von den anderen Ländern der SBZ abhebt. Der Norden zeichnete sich dadurch aus, daß die KPD/SED ihre Herrschaft hier besonders schnell und erfolgreich durchsetzte: Mecklenburg entließ alle Pgs schneller aus der Verwaltung als die anderen Landes- und Provinzialverwaltungen der SBZ; hier etablierte sich die Praxis der, permanenten Säuberung', bei der es weniger um das Verhalten in der Vergangenheit als um die gegenwärtige Loyalität zu Besatzungsmacht und SED ging, schon 1946, als die anderen Landesverwaltungen der SBZ noch immer mit der Entlassung ehemaliger NSDAP-Mitglieder beschäftigt waren. Bereits 1945 zeichnete sich die mecklenburgische Justiz innerhalb der SBZ durch sehr weitreichende politische und personelle Neuansätze aus. Die Großzügigkeit gegenüber den Flüchtlingen, die von der Entnazifizierung fast gar nicht betroffen wurden, gewann in Mecklenburg-Vorpommern eine besondere Relevanz, weil die Ortsfremden hier die Hälfte der Bevölkerung stellten und zahlreiche Positionen einnahmen, die infolge von Flucht oder Entnazifizierung vakant geworden waren. Diese Tendenz wurde dadurch verstärkt, daß in dem traditionell großagrarisch geprägten Land mehr als die Hälfte der landwirtschaftlichen Nutzflächen infolge der Bodenreform ihre Eigentümer wechselte, so daß keine Gemeinde und fast keine Stadt ihr altes Gesicht behielt.

Damit übernahm das stark traditionell geprägte Mecklenburg-Vorpommern eine Vorreiterrolle bei der Umgestaltung der SBZ-Gesellschaft und ihrer Einpassung ins SEDKonzept. Entscheidende Voraussetzungen für die früh etablierte Vormachtstellung der KPD waren neben der Gegenwart der Besatzungsmacht, dem großen politischen Geschick des Innenministers Warnke und den tiefgreifenden Veränderungen infolge von Kriegsende und Massenmigration auch der geringe Organisationsgrad der möglichen Opposition seitens der Sozialdemokraten und bürgerlicher Demokraten. Die problemlose Aneignung der politischen Führungsrolle durch die KPD scheint der traditionellen Schwäche der Arbeiterbewegung in Mecklenburg und Vorpommern zu widersprechen. Obwohl die Sozialisten und Kommunisten in den Industriezentren im Süden der SBZ gesellschaftlich weitaus besser verankert waren, übernahm der großagrarische Norden diese Rolle. Der entscheidende Grund hierfür lag in der reduzierten Komplexität sozialer und politischer Beziehungen in Mecklenburg: Zur Etablierung der SED-Herrschaft bedurfte es nur der wenigen effizient arbeitenden Kommunisten um Innenminister Warnke, die in kurzer Zeit alle machtpolitisch entscheidenden Apparate in ihrer Hand hielten. Im ganzen Land stand ihnen niemals ein vergleichbarer politischer Zusammenschluß entgegen. Weil alle politischen Organisationen inklusive der Kirche im ,Dritten Reich' oder durch die Alliierten aufgelöst, gleichgeschaltet, verboten oder geschwächt

\footnotetext{
${ }^{3}$ Srubar, Sozialismus, S. 418.

${ }^{4}$ Kocka, Durchherrschte, S. 547f.; Lüdtke, Helden, S. 188.
} 
worden waren, kamen als einzige mögliche Träger einer oppositionellen politischen Strategie nur die Parteien in Frage. CDU, LDP und SPD hatten sich in MecklenburgVorpommern 1945 aber in die vorgegebene Blockkonzeption hineingegründet und zeichneten sich seitdem durch eine weitgehend unwidersprochen hingenommene Subalternität gegenüber den Kommunisten aus. Konflikte mit der KPD blieben in Mecklenburg-Vorpommern fast vollständig aus. Die Sozialdemokraten profilierten sich bis zur SED-Gründung in der Landespolitik nicht als eigenständige politische Gruppierung und gingen danach entsprechend lautlos in der kommunistisch dominierten SED unter. In der CDU und LDP gab es ebenso wie in den anderen Ländern der SBZ eine unterschiedlich weitgehende Anpassungsbereitschaft; in Schwerin entschieden sich die schwachen Landesleitungen in Konfliktsituationen jedoch gegen ihre Parteifreunde, die einen eigenständigen oder sogar oppositionellen Kurs verfolgten, und paßten sich der hegemonialen Partei und Besatzungsmacht an. Durch Verfassung und Blocksystem fest etabliert, spielten sie damit in der Landespolitik eine ähnlich marginale Rolle wie der sozialdemokratische Ministerpräsident Höcker, dessen politischer Einfluß umgekehrt proportional zu seiner offiziellen Position war.

Anders als im Süden der SBZ gab es in Mecklenburg auch keine einflußreichen ,unpolitischen' Organisationen, die eine entscheidende Rolle in der Landespolitik hätten übernehmen können: Die wirtschaftlich bedeutendste Gruppe, die Großgrundbesitzer, war infolge ihrer Flucht und durch die Bodenreform abwesend und entmachtet; auch starke Gewerkschaften, große Privatbetriebe oder andere wirtschaftliche Zusammenschlüsse fehlten, die eigene Interessen hätten formulieren und durchsetzen können. Dieser Mangel an eigenständigen politischen und sozialen Organisationen ermöglichte es den mecklenburgischen Kommunisten, die eigenen politischen Vorstellungen weit ungehinderter durchzusetzen als in den anderen Ländern der SBZ. An der Spitze der Landespolitik etablierte sich eine kleine, nur ungenügend legitimierte Gruppe und eignete sich den entscheidenden politischen Einfluß im ganzen Land an. Somit setzten die Kommunisten und die anpassungsbereite politische Klasse 1945 die Geschichte des bis 1918 ständisch regierten, durch demokratisch-partizipative Traditionen wenig ausgezeichneten und obrigkeitsstaatlich orientierten Mecklenburgs fort. 
